\title{
Internal Force Compensating Method for Wall-Climbing Caterpillar Robot
}

\author{
W. Wang, K. Wang, H. X. Zhang, J. W. Zhang
}

\begin{abstract}
The redundant driving problem is an inherent phenomenon existing in a modular caterpillar robot. To limit the internal forces arising from the redundant driving, this paper proposes a joint torque control method, which is based on an assumption that there is only one active joint in the four-link mechanism driving the climbing gait. Except the active joint, the other three joints are all considered as passive joints, whose torques tend to be zero, although they are driven by motors in reality. According to the analyses of static forces in the closed chain state, the ideal torque of named active joint is calculated, and will be followed by the joint in real climbing locomotion. The experiments reveal the reasonability and feasibility of the proposed joint control method, as well as the limitations of current prototype and control algorithm.
\end{abstract}

\section{INTRODUCTION}

$\mathrm{M}$ ini-climbing robots will be found as useful and safe tools in the infrastructures inspection, urban search, civil exploration, etc. [1] [2], because of its portability, low-cost and less potential dangers. Up to date, there are some new tendencies in the climbing robot research. The first is the modular structure [3] [5], which enable the researchers to optimize the robot configuration by combining the modules in different ways. The second is the biologically inspired design. Mini-Whegs B00 [4] mimics the structure of a cockroach to realize the transfer between two planes. In Ref. [5], the leg motion of RiSE follows the climbing gait of the gecko. The third is the new attaching methods. In Ref. [6], Waalbot applies the nano material to attach on solid surfaces by virtue of the Van Der Waals force, which is believed the main attaching force used by the gecko. The foot of RiSE [5] is composed of some micro needles, by which the robot can climb on very rough concrete walls. The

This project is supported by the "National Natural Science Foundation of China" (No. 50605001).

W. Wang works with the Robotics Institute, School of Mechanical Engineering and Automation, Beijing University of Aeronautics and Astronautics, 37 Xueyuan Road, 100191, Beijing, China. (as the corresponding author, e-mail: wangweilab@buaa.edu.cn,).

K. Wang works with the Robotics Institute, School of Mechanical Engineering and Automation, Beijing University of Aeronautics and Astronautics, 37 Xueyuan Road, 100191, Beijing, China.

H. X. Zhang works with the Institute of Technical Aspects of Multimodal Systems, Department of Computer Science, University of Hamburg, Vogt-Koelln-Strasse 30, 22527, Hamburg, Germany (e-mail: hzhang@informatik.uni-hamburg.de,hxzhang@ieee.org).

J. W. Zhang works with the Institute of Technical Aspects of Multimodal Systems, Department of Computer Science, University of Hamburg, Hamburg, Germany(e-mail: zhang@informatik.uni-hamburg.de). fourth is the extended body length. In Ref. [5] and Ref. [6], RiSE and Waalbot apply the tails to extend their lengths to withstand the pitch-back moment, which is the most dangerous load for wall climbing robots.

Biologically inspired design may be an efficient way to find a kinematics structure for climbing robots which can compromise between the weight and flexibility. The biologists pointed out that the caterpillar is among the most successful climbers in nature, because of its available multi gaits, flexible body structure and simple locomotion control mechanism [7] [13]. From the robotics point of view, the caterpillars inspire us in the following aspects.

1) The multi-joints configuration of a caterpillar-like robot will ensure the high flexibility.

2) When a caterpillar climbing, its weight is distributed to many joints, and its center of gravity is gradually lifted. As a result, the load on one joint is decreased, and that make it possible to lighten the robot by simulating the structure of a caterpillar.

3) The caterpillar, which is composed of many segments, will be a good model for the modular design.

4) Compared with other animals, the caterpillar has a large ratio of length to height. This character will endow a caterpillar climbing robot with a large ratio of length to pitch-back moment.

5) It has been testified that the locomotion of a caterpillar is controlled by its Central Pattern Generator, which is composed of low-level motion control neuro-units. The motion control mechanism of CPG will inspire the researchers to try some biological control algorithm in the future.

Our previous work indicates that a changing kinematics chain is involved when a modular caterpillar robot crawls on walls [1]. When the robot moves in the closed chain state, a four-link model is applied to transfer the locomotion wave along the robot's body. However, the redundant driving property of the closed chain may result unexpected internal forces to slip the sucking cups of robot. To avoid this problem, the driving torques of joints in the closed chain should be further controlled.

This paper is organized as follows. Section II gives an introduction of the mechanical structure of the modular caterpillar robot and one of its climbing gaits. Section III presents a joint control method to resolve the redundant driving problem. Based on a revised four-link model, the static forces acting on the links and joints in the closed chain 
are analyzed. Furthermore, this paper proposed an assumption that only one joint drives the four-link mechanism to move, but zero torques are output by the other three joints. According to this assumption, the torque of the active joint is calculated. Section IV testifies the analyses and assumption by experiments, as well as the limitations of the proposed method. At last, the conclusions and future work are given.

\section{Overview OF Robot STRUCTURE AND Climbing GAIT}

A typical wall-climbing caterpillar robot, as shown in Fig.1, is composed of four adhesion modules and four link modules. All of these modules are connected together by motorized joints one by one. The adhesion modules are able to attach on glass, wood or flat concrete wall by virtue of a vibrating attaching method [14], which is out of scope of this paper, and will not be discussed at here.

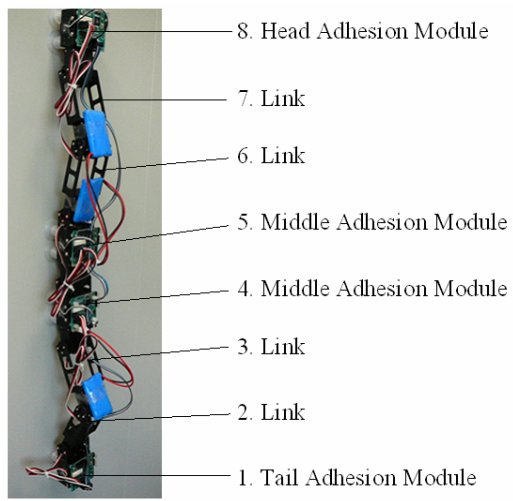

Fig. 1. A wall-climbing caterpillar robot

Each joint is driven by one servo motors (Model: hangood HG14-M), and one DC motor is installed in the adhesion module to attach the sucking cups on or release them from the wall. Table I describes some important parameters of the caterpillar robot.

TABLE I

Parameters of caterpillar robo

\begin{tabular}{lc}
\hline \multicolumn{1}{c}{ Parameters } & Value \\
\hline Whole length of the robot $L /(\mathrm{mm})$ & 750 \\
Rated torque of active driving joint $M /(\mathrm{kg} \cdot \mathrm{cm})$ & 14 \\
Angle of active driving joint $\phi_{\mathrm{i}} /\left({ }^{\circ}\right)$ & \pm 85 \\
Length of tail adhesion module (link 1) $L_{1} /(\mathrm{mm})$ & 70 \\
Length of head adhesion module (link 8) $L_{8} /(\mathrm{mm})$ & 80 \\
Length of middle adhesion module (link 2 7) $L_{2} \sim L_{7} /(\mathrm{mm})$ & 100 \\
Mass of tail adhesion module (link 1) $m_{1} /(\mathrm{g})$ & 112 \\
Mass of head adhesion module (link 8) $m_{8} /(\mathrm{g})$ & 172 \\
Mass of middle adhesion module $\left(\right.$ link 4 5) $m_{4}, m_{5} /(\mathrm{g})$ & 177 \\
Mass of link 2, link 3, link 6 and link 7 $m_{2}, m_{3}, m_{6}, m_{7} /(\mathrm{g})$ & 90 \\
\hline
\end{tabular}

Fig. 2 shows one possible safe climbing gait adopted by the caterpillar robot moving on a vertical. In which $\lambda$ is the step length, $\varphi_{0}$ is the original angle of the closed chain. From $t_{0} \sim t_{1}$ and $t_{9} \sim t_{10}$, the robot moves in open chain states, in which only three joints turn independently. Obviously, period $t_{1} \sim t_{9}$ relates to the closed chain state, in which there are four joints turning simultaneously. A four-link model was be used to denote the kinematics of the closed chain [1], by which the rotation angles of the four active joints can be determined. Besides the joint angles, the torques of joint motors are also important parameters needing identification, since improper joint torques may induce unexpected internal forces and slip the attaching sucking cups in such a redundant driving mechanism.

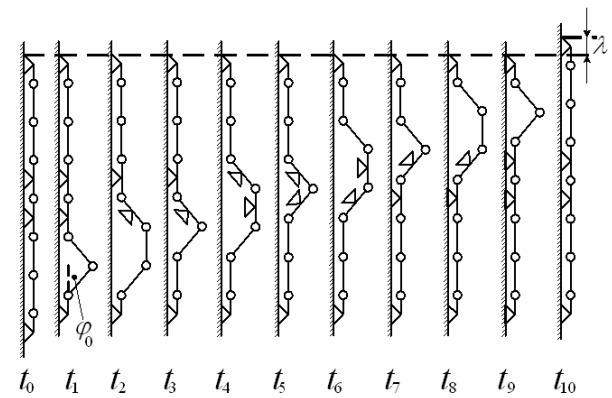

Fig. 2. Gait of wall-climbing caterpillar robot

\section{ANALYSES OF JOINT TORQUES}

A. A revised kinematics of a four-link mechanism

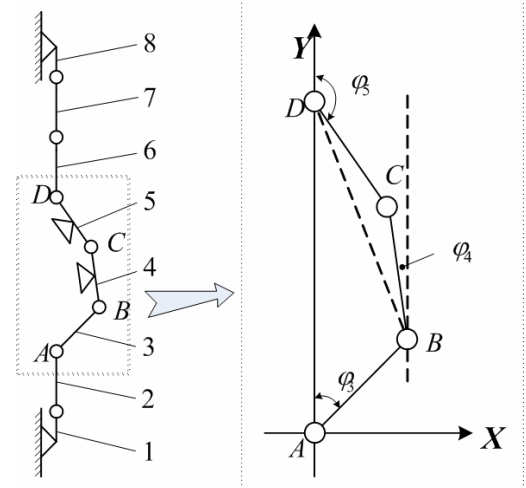

Fig. 3. A typical instant of closed chain state

Fig. 3 shows a typical instant in the closed chain state, where the turning joints are numbered $A, B, C$ and $D$. A inertial reference frame $I=\{A, X, Y\}$ is fixed with the sucking cup. Unlike our previous work in which the joint angles were considered as the parameters of a four-link mechanism, this paper takes into account the angles between three moving links and $Y$ axis. According to the geometric relation of the links, the following equations can be deduced. 


$$
\begin{aligned}
& \left\{\begin{array}{c}
l \cos \varphi_{3}=y_{B} \\
l \sin \varphi_{3}=x_{B} \\
l \cos \varphi_{4}=y_{C}-y_{B} \\
l \sin \varphi_{4}=x_{C}-x_{B} \\
l \cos \varphi_{5}=y_{C}-\tilde{l} \\
l \sin \varphi_{5}=x_{C}
\end{array}\right. \\
& \left\{\begin{array}{l}
\omega_{4}=\frac{-v_{B x} x_{C}-v_{B y}\left(y_{C}-\tilde{l}\right)}{x_{C}\left(y_{C}-y_{B}\right)-\left(y_{C}-\tilde{l}\right)\left(x_{C}-x_{B}\right)} \\
\omega_{5}=\frac{-v_{B x}\left(x_{C}-x_{B}\right)-v_{B y}\left(y_{C}-y_{B}\right)}{x_{C}\left(y_{C}-y_{B}\right)-\left(y_{C}-\tilde{l}\right)\left(x_{C}-x_{B}\right)}
\end{array}\right. \\
& \left\{\begin{array}{l}
\varepsilon_{4}=\frac{E \cdot\left(y_{C}-\tilde{l}\right)+F \cdot x_{C}}{\left(x_{C}-x_{B}\right)\left(y_{C}-\tilde{l}\right)-x_{C}\left(y_{C}-y_{B}\right)} \\
\varepsilon_{5}=\frac{E \cdot\left(y_{C}-y_{B}\right)+F \cdot\left(x_{C}-x_{B}\right)}{\left(x_{C}-x_{B}\right)\left(y_{C}-\tilde{l}\right)-x_{C}\left(y_{C}-y_{B}\right)}
\end{array}\right.
\end{aligned}
$$

Where,

$l$ is the length of links, and $A B=B C=C D=l$, $\tilde{l}=A D=\left(1+2 \cos \varphi_{0}\right) l$. When $\varphi_{0}$ is known, the values of $x_{\mathrm{b}}$, $y_{\mathrm{b}}, x_{\mathrm{c}}$ and $y_{\mathrm{c}}$ are all determined;

$\omega_{4}, \omega_{5}, \varepsilon_{4}, \varepsilon_{5}$ are the angular velocities and accelerations of links 4 and 5 respectively, and

$$
\begin{aligned}
& E=-a_{B x}-\omega_{4}^{2}\left(y_{C}-y_{B}\right)+\omega_{5}^{2}\left(y_{C}-\tilde{l}\right) ; \\
& F=-a_{B y}+\omega_{4}^{2}\left(x_{C}-x_{B}\right)+\omega_{5}^{2} x_{C} ; \\
& v_{B x}=\omega_{3} l \cos \varphi_{3}=\omega_{3}\left(y_{B}-y_{A}\right) ; \\
& v_{B y}=-\omega_{3} l \sin \varphi_{3}=-\omega_{3}\left(x_{B}-x_{A}\right) ; \\
& a_{B x}=-\omega_{3}^{2}\left(x_{B}-x_{A}\right)+\varepsilon_{3}\left(y_{B}-y_{A}\right) ; \\
& a_{B y}=-\omega_{3}^{2}\left(y_{B}-y_{A}\right)-\varepsilon_{3}\left(x_{B}-x_{A}\right) ;
\end{aligned}
$$

\section{B. Driving torque of active joint}

To limit the unexpected internal forces arisen from the errors of joint control or the inaccuracy of mechanical structure, only one joint is considered as an active joint, and the other three joints follows the active joint to turn, though these four joints are all driven by motors simultaneously during the closed chain state. If an assumption is taken into consideration that the ideal joint torques of the passive joints all tend to be zero, the active joint will be responsible for driving the four-link mechanism to transfer the locomotion wave along the robot body. It is acceptable to choose the joint which is closest to the tail as the active joint. The following analysis indicates the desired torque of the active joint.

Fig. 4 shows the static forces acting on the robot when climbing in the closed chain state. In which, $L_{\mathrm{s}}$ is the height of the sucking cup; $Q\left(Q_{\mathrm{x}}, Q_{\mathrm{y}}\right)$ is the instantaneous center of gravity of the robot; $f_{1}$ and $f_{8}$ are the friction forces between sucking cup 1, 8 and the wall respectively; $N_{1}$ and $\mathrm{N}_{8}$ are the supporting forces of the wall to cup 1 and 8 , which can be indicated by equations (4).

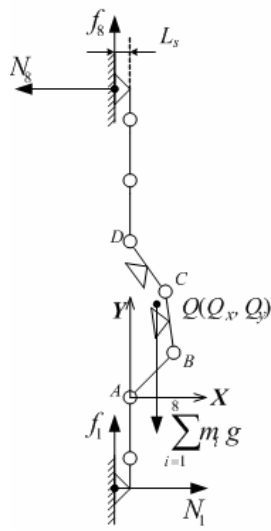

Fig. 4. Static forces on robot

$$
N_{1}=N_{8}=\frac{Q_{x}+L_{s}}{4 l+\tilde{l}} \cdot \sum_{i=1}^{8} m_{i} g
$$

Where,

$m_{\mathrm{i}}$ is the mass of one module;

$$
Q_{x}=\frac{\sum_{i=1}^{8} Q_{i x} m_{i}}{\sum_{i=1}^{8} m_{i}}=\frac{m_{3} k_{3} x_{B}+m_{4}\left[\left(1-k_{4}\right) x_{B}+k_{4} x_{C}\right]+m_{5}\left(1-k_{5}\right) x_{C}}{\sum_{i=1}^{8} m_{i}} ;
$$

$k_{\mathrm{i}}$ is a constant representing the position of the center of gravity of one link;

The coordinate values of $\mathrm{B}$ and $\mathrm{C}$, namely $x_{\mathrm{B}}, y_{\mathrm{B}}, x_{\mathrm{C}}$, and $y_{\mathrm{C}}$, can be calculated according to $\varphi_{0}, \varphi_{3}$, and $l$.

To identify the theoretic driving torque of the active joint, the forces acting on the turning joints and the moving links should be known.

Fig. 5 shows the forces on link 6, namely $F_{x}{ }^{(56)}$ and $F_{y}{ }^{(56)}$, which can be expressed by equations (5) and (6). The forces on link 5 , link 4 and link 3 are respectively denoted by Fig. 6 , Fig. 7, Fig. 8 and equations (7) (11).

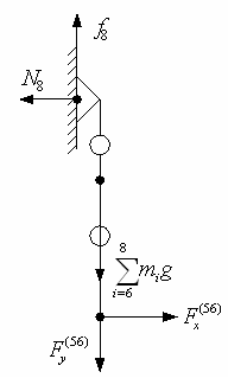

Fig. 5. Mechanical model of link 6, link 7 and link 8 


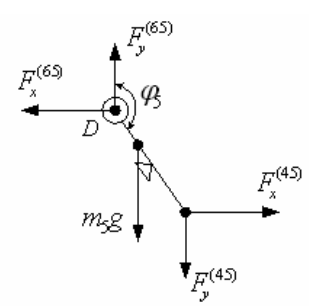

Fig. 6. Mechanical model of link 5

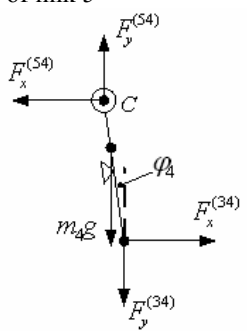

Fig. 7. Mechanical model of link 4

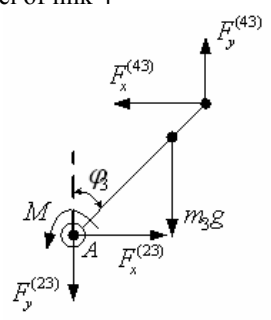

Fig. 8. Mechanical model of link 3

$$
\begin{aligned}
F_{x}^{(56)}= & \frac{Q_{x}+L_{s}}{4 l+\tilde{l}} \cdot \sum_{i=1}^{8} m_{i} g \\
F_{y}^{(56)}= & f_{8}-\sum_{i=6}^{8} m_{i} g=\frac{1}{2} \sum_{i=1}^{8} m_{i} g-\sum_{i=6}^{8} m_{i} g \\
F_{x}^{(45)}= & \frac{m_{5} g k_{5} x_{C} \cos \varphi_{5}}{x_{C} \sin \varphi_{5}+\left(y_{C}-\tilde{l}\right) \cos \varphi_{5}} \\
& -\frac{m_{5} l\left(1-k_{5}\right)\left[\left(y_{C}-\tilde{l}\right)\left(1-k_{5}\right) \varepsilon_{5}+x_{C} \omega_{5}^{2}\right]}{x_{C} \sin \varphi_{5}+\left(y_{C}-\tilde{l}\right) \cos \varphi_{5}} \\
& +\frac{F_{x}^{(65)} x_{C} \sin \varphi_{5}-F_{y}^{(65)} x_{C} \cos \varphi_{5}}{x_{C} \sin \varphi_{5}+\left(y_{C}-\tilde{l}\right) \cos \varphi_{5}} \\
F_{y}^{(45)}= & -\frac{m_{5} g\left[\left(y_{C}-\tilde{l}\right) \cos \varphi_{5}+\left(1-k_{5}\right) x_{C} \sin \varphi_{5}\right]}{x_{C} \sin \varphi_{5}+\left(y_{C}-\tilde{l}\right) \cos \varphi_{5}} \\
& +\frac{m_{5} l\left(1-k_{5}\right)\left[\left(y_{C}-\tilde{l}\right) \omega_{5}^{2}-\left(1-k_{5}\right) x_{C} \varepsilon_{5}\right]}{x_{C} \sin \varphi_{5}+\left(y_{C}-\tilde{l}\right) \cos \varphi_{5}} \\
F_{x}^{(34)}= & \frac{\left(y_{C}-\tilde{l}\right)\left[F_{y}^{(65)} \cos \varphi_{5}-F_{x}^{(65)} \sin \varphi_{5}\right]}{x_{C} \sin \varphi_{5}+\left(y_{C}-\tilde{l}\right) \cos \varphi_{5}} \\
& \left(x_{C}-x_{4} g k_{4}\left(x_{C}-x_{B}\right) \sin \varphi_{4}+\left(y_{C}-y_{B}\right) \cos \varphi_{4}\right. \\
+ & \frac{m_{4} l\left(1-k_{4}\right)\left[\left(x_{C}-x_{B}\right) \varpi_{4}^{2}+g\left(1-k_{4}\right)\left(y_{C}-y_{B}\right) \varepsilon_{4}\right]}{\left(x_{C}-x_{B}\right) \sin \varphi_{4}+\left(y_{C}-y_{B}\right) \cos \varphi_{4}} \\
& +7) \\
&
\end{aligned}
$$


joint torque is controlled in real time according to the ideal joint value deduced from equation (11). A series of experiments are performed on vertical glass panes. A complete period of crawling is shown in Fig. 10, in which the photos are just corresponding to the $t_{0} \sim t_{10}$ steps in Fig. 2. Some basic experiment results are shown in Table II.

TABLE II

Results of wall-climbing experiment

\begin{tabular}{lc}
\hline \multicolumn{1}{c}{ Parameters } & Value \\
\hline Power consumption $P /(\mathrm{W})$ & About 8 \\
Climbing velocity $v /(\mathrm{mm} / \mathrm{min})$ & 67.5 \\
Initial angle $\phi_{0} /\left(^{\circ}\right)$ & 30 \\
Step length $\lambda /(\mathrm{mm})$ & 27 \\
\hline
\end{tabular}

If the force control is not mixed with the position control, the robot may suffer from unforeseen slip of the sucking cups, which sometimes results the failures of climbing, such as falling down from the glass, less step length, etc. On the contrary, a smooth climbing locomotion can be realized reliably when the joint forces are compensated properly.

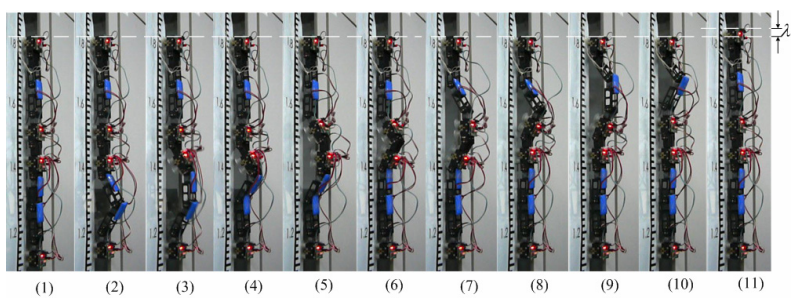

Fig. 10. The experimental photos of caterpillar robot

However, the experiments also reveal the problem of current prototype and gait control method. The passive joints can't be completely regarded as hinges, because it is impossible to restrain their joint torques to zero completely. As a result, slight fluctuations of the joint angles may occur sometimes.

\section{CONCLUSIONS}

Based on the above analyses and experiments, this paper yields the following conclusions:

1) Although the four-link mechanism of a modular caterpillar robot simplifies the gait control, it suffers from the redundant driving phenomenon because of its over-abundance actuators.

2) To overcome the redundant driving problem, the torque control is necessary to limit the internal forces.

3) When the force control algorithm is taken into account, it is reasonable to assume that only one joint is the active joint and the other joints are passive ones, whose torques tends to be zero.

In the future, the researcher will focus on improving the control precision of joint force compensation for the passive joints and optimizing the algorithm. A compliant structure will be another choice to resolve the redundant driving problem existing in the caterpillar climbing robot.

\section{REFERENCES}

[1] W. Wang, H.X. Zhang, Y.Y. Wang, et al., "Analysis of the kinematics of module climbing caterpillar robots", Proceeding of 2008 IEEE/ASME International Conference on Advanced Intelligent Mechatronics. Xi'an, China, June 4-7, 2008, pp. 84-89.

[2] Wu SQ, Li MT, Xiao S, et al. A wireless distributed wall climbing robotic system for reconnaissance purpose. In: Proceedings of the 2006 IEEE International Conference on Mechatronics and Automation. Luoyang, China, June 12-15, 2006, p. 1380-1312.

[3] Tlale NS. A modular design of a wall-climbing robot and its mechatronics controller. Journal of Industrial Engineering 2006; 17(2): 197-208.

[4] Daltorio KA, Witushynsky TC, Wile GD, et al. A body joint improves vertical to horizontal transitions of a wall-climbing robot. In: Proceedings of 2008 IEEE International Conference on Robotics and Automation. Pasadena, CA, USA, May 19-23, 2008, p. 3046-3051.

[5] Spenko MJ, Haynes GC, Saunders JA, et al. Biologically inspired climbing with a hexapedal robot. Journal of Field Robotics 2008; 25(4-5): 223-242.

[6] Aksak B, Murphy MP, and Sitti M. Gecko inspired micro-fibrillar adhesives for wall climbing robots on micro/nanoscale rough surfaces. In: Proceedings of 2008 IEEE International Conference on Robotics and Automation Pasadena. CA, USA, May 19-23, 2008, p. 3058-3063.

[7] Brackenbury J. Caterpillar kinematics. NATURE 1997; 390(4 December): 453.

[8] Trimmer B, Issberner J. Kinematics of soft-bodied, legged locomotion in manduca sexta larvae. Journal of Experimental Biology 2007; 212 (3): $130-142$.

[9] Dickinson MH, Farley CT, Full RJ, et al. Movement: molecular to robotic, review - how animals move: an integrative view. SCIENCE 2000; 288(7 April): 100-106.

[10] Brackenbury J. Fast locomotion in caterpillars. Journal of Insect Physiology 1999; 45 (5): 525-533

[11] Woods WA, Fusillo SJ, Trimmer BA. Dynamic properties of a locomotory muscle of the tobacco hornworm Manduca sexta during strain cycling and simulated natural crawling. Journal of Experimental Biology 2008; 211(1): 873-882.

[12] Berlanger JH, Trimmer BA. Combined kinematic and electromyographic analyses of proleg function during crawling by the caterpillar Manduca sexda. Journal of Comparative Physiology A: Neuroethology, Sensory, Neural, and Behavioral Physiology 2002; 186(11): 1031-1039.

[13] Trimmer BA, Takesian AE, Sweet BM, et al. Caterpillar locomotion: a new model for soft-body climbing and burrowing robots. In: Proceedings of 7th International Symposium on Technology and the Mine Problem. Monterey, CA, May 2-5, 2006, p. 1-10.

[14] Kun Wang, Wei Wang, Dazhai Li, Guanghua Zong, Houxiang Zhang, Jianwei Zhang, Zhicheng Deng, Analysis of two vibrating suction methods, 2008 IEEE International conference on Robotics and Biomimetics (ROBIO 2008), Bangkok, Thailand, February 21-26, 2009, pp. 1313-1318 